\section{Modificación de estereotipos negativos en la vejez en estudiantes de enfermería}

\section{Changing negative stereotypes in old age in nursing students}

Carmen María Sarabia Cobo ${ }^{1}$

Cristina Castanedo Pfeiffer ${ }^{2}$

1. Ayudante LOU, área Enfermería del Envejecimiento, Departamento de Enfermería, Universidad de Cantabria. Santander.

2. Profesora titular, área Enfermería del Envejecimiento, Departamento de Enfermería, Universidad de Cantabria. Santander.

\section{Correspondencia:}

Dra. Carmen María Sarabia Cobo

EUE Casa de Salud Valdecilla

Avda. Valdecilla, s/n

39011 Santander

E-mail: carmen.sarabia@unican.es

\section{RESUMEN}

El objetivo de este estudio fue explorar la modificación de los estereotipos y mitos sobre la vejez en estudiantes de tercer curso del Grado en Enfermería antes y después de impartir la asignatura Enfermería del Envejecimiento.

Método: se realizó un estudio descriptivo intrasujeto de medidas repetidas. Se empleó el Cuestionario de estereotipos negativos hacia la vejez (CENVE).

Resultados: la prevalencia del estereotipo negativo global fue del $62,0 \%$ en la medida preintervención (M1) y del 12,3\% en la postintervención (M2); para el factor salud, del 63,5\% (M1) y del 9,2\% (M2); para motivacional-social, del 43,1\% (M1) y del 4,9\% (M2), y para carácter-personalidad, del 58,3\% (M1) y del 3,8\% (M2). Se realiza la prueba de $t$ de Student apareada que confirma que las diferencias son estadísticamente significativas.

Conclusiones: existe una prevalencia alta de estereotipos negativos hacia la vejez entre los estudiantes de enfermería a pesar de haber realizado prácticas clínicas y estar en el tercer curso del Grado en Enfermería. La asignatura ha demostrado ser eficaz para la modificación de estos estereotipos. La adecuada formación de los futuros profesionales contribuirá de manera notoria a la dispensación de un cuidado adecuado, erradicando tendencias como el edadismo que siguen siendo prevalentes en el sistema sanitario.

PALABRAS CLAVE: estereotipo, edadismo, envejecimiento, enfermería, actitud del personal sanitario.
The aim of this study was to explore the modification of stereotypes and myths about aging in nursing degree students before and after teaching the subject Nursing Aging.

Method: The research was a descriptive study within-subject repeated measures. It was used the Questionary Aging Negative Stereotypes (CENVE).

Results: The overall prevalence of negative stereotypes was $62.0 \%$ in the pre-intervention measure (M1) and $12.3 \%$ in the postintervention (M2), for the health factor, $63.5 \%$ (M1) and 9.2\% (M2), for social reasons, $43.1 \%$ (M1) and 4.9\% (M2) and personality character, 58.3\% (M1) and 3.8\% (M2). Is performed paired Student $t$ confirming that the differences were statistically significant.

Conclusions: There is a high prevalence of negative stereotypes toward the elderly among nursing students despite having done clinical practice and be in third grade. The subject has proven effective for changing these stereotypes. Proper training of future professionals contribute markedly to the dispensation of proper care, eradicating ageism trends as they are still prevalent in the healthcare system.

KEYWORDS: stereotype, ageism, aging, nursing, attitude of health personnel.

\section{- INTRODUCCIÓN}

Según el Plan de Acción Internacional de Madrid sobre el envejecimiento (2002), para mediados del siglo $\mathrm{xxI}^{1}$, la proporción de mayores y jóvenes será la misma, de tal forma que el número de mayores de 60 años se duplicará para el año 2050. Es un hecho que estamos asistiendo al envejecimiento de la población, a un considerable aumento de las enfermedades crónicas y de los estados de dependencia y atención al mayor, así como a la búsqueda de políticas que favorezcan el envejecimiento activo. Sabemos que el sistema sanitario y el social atienden mayoritariamente y cada vez más a población mayor.
Según Barón y Byrne ${ }^{2}$, los estereotipos son creencias referidas a características o rasgos compartidos por miembros de grupos sociales específicos y los rasgos típicos o modales que supuestamente poseen quienes pertenecen a dichos grupos. Las ideas o creencias sobre el proceso de envejecimiento y la vejez, cuando suponen connotaciones de valoración positiva o negativa, se convierten en actitudes o prejuicios que pueden favorecer la aparición de conductas discriminatorias hacia los miembros de un determinado grupo. La discriminación basada en la edad da lugar a conductas y situaciones en las que los ancianos reciben un trato peor o diferente por el hecho de ser mayores. Según Blanca, Sánchez y Trianés ${ }^{3}$, los estereotipos negativos y mitos en torno a la vejez se basan en la creencia de la incapacidad física y psíquica y la enfermedad como 
sinónimo de vejez, y aunque los estudios sobre estereotipos en la vejez aún son escasos, la mayoría confirman esos aspectos.

Por tanto, envejecer en nuestra sociedad tiene connotaciones negativas que se asocian a decadencia y carga, destacando el carácter biológico de deterioro y pérdida de capacidades ${ }^{4}$, y estos estereotipos negativos son mantenidos tanto por la sociedad como por el grupo de edad5.

El término edadismo fue acuńado para referirse al concepto peyorativo de alguien por tener una edad avanzada ${ }^{6}$. Dicho término es fruto de estereotipos negativos vinculados a la vejez, así como mitos que la sociedad crea entorno a esta etapa. Diversas disciplinas como la Psicología o la Sociología se han interesado por el estudio de los estereotipos y prejuicios que la sociedad tiene de la vejez. Es cierto que esta realidad sobre la existencia de estereotipos negativos hacia la vejez se viene investigando desde hace cinco décadas, pero ha sido hace relativamente poco cuando se han multiplicado las investigaciones con la creación de diferentes test que lo evalúan. Esto puede ser fruto del envejecimiento creciente de la población en los últimos ańos. Se han investigado tanto grupos de mayores como familiares y cuidadores (asociación de vejez y enfermedad, distorsión de la capacidad), así como los jóvenes (visión de decadencia, soledad y pérdidas) y otros grupos. En los estudios centrados en profesionales sanitarios se ha puesto de manifiesto la falta de conocimientos sobre el proceso de envejecer, la tendencia al edadismo y a justificar la patología por "propio de la edad", así como a no diagnosticar y tratar adecuadamente al mayor por el simple hecho de serlo. También se asocia a la edad los conceptos de fragilidad y debilidad. Y también se ha observado que una mayor relación profesional-paciente no disminuye estas concepciones negativas. Según Losada-Baltar ${ }^{7}$, este término, edadismo, da lugar, en la práctica profesional, a prácticas discriminatorias.

Atendiendo a lo expuesto hasta ahora cabe por tanto destacar que cada vez será mayor el número de profesionales sanitarios vinculados a la atención gerontológica en los años venideros. Partiendo del hecho de que estudios previos ponen de manifiesto la existencia de estereotipos negativos hacia la vejez, cabe plantearse desde las universidades una formación integral y adecuada de los futuros profesionales que destierre dichos mitos. Atendiendo a que la presencia de creencias erróneas y negativas puede dar lugar a prácticas discriminatorias entre los profesionales, es una responsabilidad educativa dotar a los estudiantes de conocimientos y habilidades y, sobre todo, actitudes adecuadas hacia una atención apropiada en la vejez.

El objetivo principal de este estudio fue explorar la modificación de los estereotipos y mitos sobre la vejez en estudiantes de tercer curso del Grado en Enfermería antes y después de impartir la asignatura Enfermería del Envejecimiento.

\section{- MATERIAL Y MÉTODOS}

Se realizó un estudio descriptivo intrasujeto de medidas repetidas (pre- y postintervención) en estudiantes de tercer curso del Grado en Enfermería de la Escuela Universitaria de Enfermería de la Universidad de Cantabria $(n=76)$.

Instrumento. Para la exploración de estereotipos negativos sobre la vejez se empleó el Cuestionario de estereotipos negativos hacia la vejez (CENVE) ${ }^{3}$, formado por 15 ítems, que explora tres factores: salud, motivacional-social y carácter-personalidad, compuesto por cinco ítems cada uno y cuyo formato de respuesta sigue un modelo de tipo Likert de cuatro valores. En el factor salud se explora acerca del deterioro de la salud, aparición de discapacidades, deterioro cognitivo y la existencia generalizada de enfermedades mentales en la vejez; en el factor motivacional-social, los ítems se relacionan con las carencias afectivas, falta de intereses vitales y capacidad disminuida para desempeñar una actividad laboral, y en el factor carácter-personalidad se hacen afirmaciones sobre los problemas de labilidad emocional y cambios en la personalidad. La puntuación del instrumento oscila entre 5 y 20 puntos para cada uno de los tres factores. La máxima puntuación del cuestionario es de 60 puntos, y la puntuación para cada dimensión está entre 5 y 20 puntos. La interpretación de los resultados del total del cuestionario es la siguiente: a) de 15 a 28 puntos corresponde a muy bajo, b) de 29 a 39 equivale a bajo, c) de 40 a 50 corresponde a alto y d) de 51 a 60 equivale a muy alto nivel de estereotipo negativo hacia las personas mayores.

Dicho cuestionario se pasó el primer día de impartición de la asignatura Enfermería del Envejecimiento, en formato papel, que rellenaron todos los alumnos de forma anónima. Tras los tres meses y medio de docencia, el último día de clase los estudiantes rellenaron de nuevo el test. Es interesante indicar que los estudiantes de tercero ya habían realizado prácticas clínicas en centros sociosanitarios, en hospitales y centros de salud, pero no habían recibido formación específica en gerontología. Se obtuvo autorización por escrito de la Dirección de la Escuela para llevar a cabo el estudio. Asimismo, los estudiantes accedieron libremente a responder al cuestionario en las dos ocasiones, tras dar su consentimiento verbal. Para el análisis estadístico se empleó el software IBM SPSS Statistics 20. Se adoptó un nivel de confianza del 95\% (nivel de significación $\alpha=0,05$ ).

\section{- RESULTADOS}

La edad promedio de la población estudiada es de 20,37 años; predomina el sexo femenino $(88,4 \%)$. El pretest (M1) lo contestaron 77 estudiantes y el postest (M2), 74. La prevalencia del prejuicio de categorías "algo de acuerdo" y «muy de acuerdo" en el factor salud es del 46,5\% y $18,0 \%$, respectivamente para M1, y del $21,3 \%$ y $2,8 \%$ para M2. Para el factor motivacional-social, del $41,8 \%$ y $12,0 \%$ en $\mathrm{M} 1$, y $12,3 \%$ y $0 \%$ en M2. Y para el factor carácter-personalidad, del 38,3\% y 19,2\% en M1, y $9,2 \%$ y $1,2 \%$ en $\mathrm{M} 2$.

La prevalencia del estereotipo negativo global es del 62,0\% (M1) y del 12,3\% (M2); para el factor salud, del 63,5\% (M1) y del 9,2\% (M2); para motivacional-social, del 43,1\% (M1) y del 4,9\% (M2), y para carácter-personalidad del 58,3\% (M1) y del 3,8\% (M2) (tabla 1).

Para comprobar si las diferencias pre- y post- son significativas, se realizó la $t$ de Student apareada, la cual confirmó diferencias significativas para los tres factores así como para la puntuación global $(p<0,005$ en todos los casos). Esto confirma que la intervención (asignatura Enfermería del Envejecimiento) fue eficaz en la modificación de estereotipos negativos.

Tabla 1. Distribución de frecuencias del estereotipo negativo por dimensión y global

\begin{tabular}{|l|c|c|}
\hline & M1 & M2 \\
\hline Estereotipo negativo global & $62 \%$ & $12,3 \%$ \\
\hline Factor salud & $63,5 \%$ & $9,2 \%$ \\
\hline Factor motivacional-social & $43,1 \%$ & $4,9 \%$ \\
\hline Factor carácter-personalidad & $58,3 \%$ & $3,8 \%$ \\
\hline
\end{tabular}




\section{- DISCUSIÓN}

El estereotipo hacia la vejez es cultural y, por ende, forma parte de la sociedad $^{8,9}$. Los resultados M1 del estudio están en consonancia con los estudios sobre estereotipos de la vejez que concluyen que la percepción social sobre las personas mayores es básicamente negativa ${ }^{10}$.

Hasta la fecha, este estudio es novedoso en cuanto a que, hasta donde sabemos, es el primero en explorar la modificación de estereotipos negativos hacia la vejez en estudiantes de enfermería, que ya han rotado por el sistema sanitario en sus prácticas, tras cursar la asignatura Enfermería del Envejecimiento. Resulta llamativo que los estudiantes mantengan niveles altos de estereotipos negativos hacia la vejez estando en tercer curso, lo que sugiere que en parte por su propio bagaje y en parte por la influencia del sistema sanitario en el que han rotado, pueden ser responsables de ello. Esto parece corroborarse en estudios realizados con personal médico ${ }^{9,11}$. Si a esto le sumamos que en la literatura especializada se ha descrito que el estereotipo negativo en el personal médico se asocia con la calidad de la atención ${ }^{12}$, es más que destacable la incorporación de formación específica en gerontología que trabaje una visión integral del mayor. Dentro de la asignatura Enfermería del Envejecimiento y como competencia específica se encuentra favorecer actitudes positivas y adecuadas en el cuidado al mayor. Sabemos que el aprendizaje se basa en el modelo de tríada: conocimientos, habilidades y actitudes. Si lo último influye en los dos primeros a la hora de adquirir adecuadamente una materia, es muy importante desarrollar la formación universitaria trabajando la actitud del estudiante, lo que sabemos repercutirá en una mejor atención profesional en el futuro.

Por tanto, podemos concluir que la formación específica en gerontología y cuidados en la vejez es una herramienta eficaz para educar en conocimientos, habilidades y actitudes positivas hacia la vejez en los futuros profesionales de enfermería

\section{- BIBLIOGRAFÍA}

1. Plan de acción internacional Madrid. (2002). Plan de acción internacional Madrid [acceso 15 de septiembre de 2012]. Disponible en: http://www.eclac.org/celade/noticias/noticias/3/9803/Plandeaccion 2002.Pdf

2. Barón R, Byrne D. Psicología Social. España: Prentice Hall; 2005.

3. Blanca M, Sánchez C, Tríanes M. Cuestionario de evaluación de estereotipos negativos hacia la vejez. Rev Multi Geront. 2005;15 (4):212-20.

4. Moragas R. Gerontología social. Envejecimiento y calidad de vida. Barcelona: Herder; 1991.
5. Wade S. Combating ageism: An imperative for contemporary health care. Rev Clin Gerontol. 2001;11:285-94.

6. Butler DM, Lewis MI. Agin and mental health: Positive psychosocial approaches. San Luis: C.V. Mosby; 1982.

7. Losada-Baltar A. Edadismo: consecuencias de los estereotipos, del prejuicio y la discriminación en la atención a las personas mayores. 2004 [acceso el 7 marzo de 2012]. Disponible en: http://envejecimiento.csic.es/documentacion/biblioteca/registro.htm?id=50818

8. Hummert ML, Garstka TA, Shaner JL, Strahm S. Judgments about stereotypes of elderly. Res Aging. 1995;2:168-89.
9. Montorio I. Actitudes hacia las personas mayores y discriminación basada en la edad. Rev Multi Geron. 1998;8:21-30.

10. Dulcey E, Ardila R. Actitudes hacia los ancianos. Rev Latino Psico. 1976:8:57-67.

11. Amy JC, Cuddy AJC, Norton MI, Fiske ST. This old stereotype: the pervasiveness and persistence of the elderly stereotype. J Social Issues. 2005;61:267-85

12. Levy BR, Banaji MR. Implicit ageism. En: Nelson T, editor. Stereotypes and prejudice against older persons. Cambridge: MIT Press 2002. p. $49-75$. 